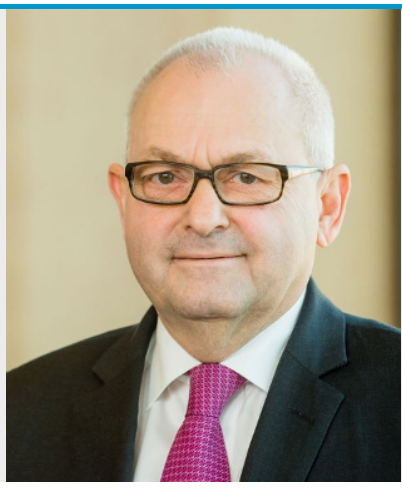

॥ Das Ziel muss weiter sein, Spitzenmedizin für die gesamte Bevölkerung zu gewährleisten

Prof. Dr. Stephan Schmitz

MVZ für Hämatologie und Onkologie Köln

Sachsenring 69, 50677 Köln, Deutschland

schmitz@oncokoeln.de

\title{
Optimale Versorgung für alle
}

Der Deutsche Krebskongress (DKK) 2020 steht unter dem Motto informativ. innovativ. integrativ. Optimale Versorgung für alle. Versorgung für alle bedeutet, dass wir Ärztinnen und Ärzte den Anspruch haben jeden Menschen mit einer Krebserkrankung in Deutschland unabhängig von seinen finanziellen Möglichkeiten, unabhängig von seiner Herkunft, unabhängig von seiner sozialen Stellung, unabhängig von seiner religiösen oder politischer Einstellung nach dem aktuellen Stand des medizinischen Wissen behandeln zu können. Das deutsche Gesundheitswesen bietet dies in großem Maße und ist eine der großen sozialen Errungenschaften unserer Gesellschaft. Dabei garantiert der Dreiklang der onkologischen Versorgung aus Universitätsklinika, aus Krankenhäusern, Praxen und Schwerpunktpraxen den sozialen barrierefreien Zugang für alle Patienten. Der medizinische Fortschritt erfordert eine zunehmende Komplexität der interdisziplinären und intersektoralen Zusammenarbeit.

Vor den zukünftigen Herausforderungen gibt es noch Optimierungspotential. Die Herausforderungen kann man unter drei Stichpunkten subsummieren: Demografie mit zunehmenden Pa- tientenzahlen, medizinischer Fortschritt und Veränderungsprozesse in der Organisation der ambulanten Leistungserbringung. Das Ziel muss weiter sein, Spitzenmedizin für die gesamte Bevölkerung zu gewährleisten. Um das gute Niveau zu halten und noch zu verbessern, sind innovative Wege notwendig. Sektorübergreifende Konzepte sind dabei eine conditio sine qua non. Dabei sollten die Werte, für die die Ärztinnen und Ärzte in Deutschland stehen, Verantwortung und Therapiefreiheit gegenüber jedem einzelnen Patienten, erhalten bleiben.

Ihr

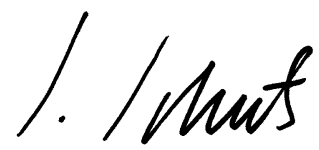

Prof. Dr. Stephan Schmitz

Schriftleiter best practice onkologie 